\title{
IDENTIFYING GENERALIZED FITZHUGH- NAGUMO EQUATION FROM A NUMERICAL SOLUTION OF HODGKIN-HUXLEY MODEL
}

\author{
NIKOLA V. GEORGIEV
}

Received 25 November 2002 and in revised form 16 January 2003

An analytic time series in the form of numerical solution (in an appropriate finite time interval) of the Hodgkin-Huxley current clamped (HHCC) system of four differential equations, well known in the neurophysiology as an exact empirical model of excitation of a giant axon of Loligo, is presented. Then we search for a second-order differential equation of generalized Fitzhugh-Nagumo (GFN) type, having as a solution the given single component (action potential) of the numerical solution. The given time series is used as a basis for reconstructing orders, powers, and coefficients of the polynomial right-hand sides of GFN equation approximately governing the process of action potential. For this purpose, a new geometrical method for determining phase space dimension of the unknown dynamical system (GFN equation) and a specific modification of least squares method for identifying unknown coefficients are developed and applied.

\section{Introduction}

Here, we experimentally base our considerations on the well-known mathematical model of nerve physiology as reported by Hodgkin and Huxley. In a series of experiments, they fixed electrodes along the entire length of a giant axon of Loligo, and the electrodes were used to measure the voltage as it varied during a depolarization event $[3,4,5,6,7]$. This is called the current clamped experimental system. Its behavior is governed by a corresponding system of four differential equations proposed by 
Hodgkin and Huxley in the form

$$
\begin{aligned}
\frac{d V}{d t} & =-\left(50+36 n^{4}(V-12)\right)-120 m^{3} h(V+115)-0.3(V+10.613), \\
\frac{d n}{d t} & =\frac{0.01(V+10)(1-n)}{\exp ((V+10) / 10-1)}-0.125 n \cdot \exp \left(\frac{V}{80}\right) \\
\frac{d m}{d t} & =\frac{0.1(V+25)(1-m)}{\exp ((V+25) / 10-1)}-4 m \cdot \exp \left(\frac{V}{18}\right) \\
\frac{d h}{d t} & =0.07(1-h) \exp \left(\frac{V}{20}\right)-\frac{h}{\exp ((V+30) / 10+1)}
\end{aligned}
$$

where $t$ is a time measured in milliseconds; from the computational point of view it is convenient to take $V$ proportional to action potential presented in scores of millivolts; $m, n$, and $h$ are empirical dimensionless variables not having clear physical sense yet. All systems (with various values of constants involved) of type (1.1) are also called Hodgkin-Huxley current clamped (HHCC) model. The concrete numerical values of constants in (1.1) are taken from [6], where they have been obtained as a result of experimental measurements and computations. In the interval $t \in(0,30)$ and at initial conditions $V=0, m=0.05, n=0.3$, and $h=0.06$, the system (1.1) has a solution whose first component $V$ is presented in Figure 1.1.

The computational neurobiology has a long history containing a huge amount of extensive studies whose review needs a special investigation. Some of them are closely related and even relevant to this work $[1,9,12]$. For example, in [9], a scheme for systematically reducing the number of differential equations required for biophysically realistic neuron models is presented. The techniques are general, are designed to be applicable to a large set of such models, and retain in the reduced system as high a degree of fidelity to the original system as possible.

In this paper, we pose the question whether or not it is possible to find a second-order differential equation of a generalized Fitzhugh-Nagumo (GFN) type

$$
\frac{d^{2} V}{d t^{2}}+P_{e}(V) \frac{d V}{d t}+P_{o}(V)+I=0
$$

having numerical solution, which is near enough to some part of the solution presented in Figure 1.1. If so, then we can talk about both qualitative and quantitative correspondences between GFN equation and HHCC model $[2,8,10]$. In $(1.2)$, the functions $P_{e}(V)$ and $P_{o}(V)$ are even 


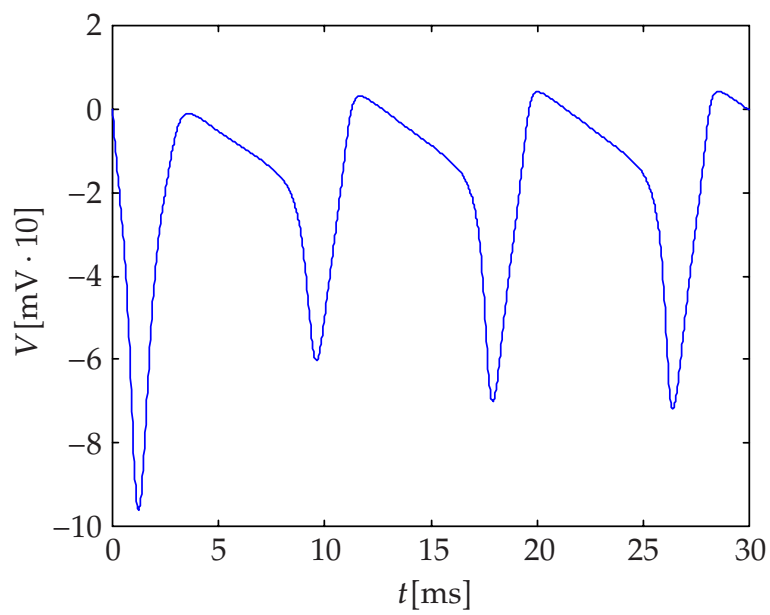

FIGURE 1.1. Action potential obtained from HHCC model in the interval $t \in(0,30)$.

and odd polynomials, respectively. Under these conditions for $P_{e}(V)$ and $P_{o}(V),(1.2)$ could be considered as a generalized Lenard equation having limit cycles in the phase-plane $(V, \dot{V})$. The constant $I$ is a membrane electrical current. The well-known particular case of Fitzhugh-Nagumo equation can be obtained from (1.2) by replacing in it the substitutions

$$
P_{e}(V)=-1+b c+V^{2}, \quad P_{o}(V)=c(1-b) V+\frac{1}{3} b c V^{3}, \quad I=a c .
$$

Here, the parameters $a, b$, and $c$ are the constants $a=0.7, b=0.8$, and $c=0.08$. The virtue of the concrete equation is in elucidating the regions of physiological behavior of axon response.

\section{Determining phase space dimension of unknown equation from a given solution}

Recently, it has been proven (see [11]) that the following theorem is valid for a given function $V(t)$.

THEOREM 2.1. Let $V(t)$ be a real-valued and analytic function defined on interval $(a, b)$ such that for every $t \in(a, b)$, the curve

$$
\vec{c}(t) \equiv\left(V(t), \frac{d V(t)}{d t}, \ldots, \frac{d^{m-1} V(t)}{d t^{m-1}}\right), \quad m>1,
$$


is simple and regular. Then there exists a unique real-valued analytic function $F_{c}\left(V, d V / d t, \ldots, d^{m-1} V / d t^{m-1}\right)$ defined on the curve $\vec{c}(t)$ such that $V(t)$ is a solution to

$$
\frac{d^{m} V}{d t^{m}}=F_{c}\left(V, \frac{d V}{d t}, \ldots, \frac{d^{m-1} V}{d t^{m-1}}\right) .
$$

In Figure 2.1, a graph of the two-dimensional curve $\vec{c}(t) \equiv\{V(t)$, $d V(t) / d t\}$ obtained by numerical differentiation of $V(t)$ is plotted. There are two points of self-intersection of the curve $\vec{c}(t)$. This means that $\vec{c}(t)$ is not simple and the well-known Cauchy theorem is not valid in the points of self-intersection. Thus $\vec{c}(t)$ is not phase trajectory of a secondorder $(m=2)$ differential equation of type (2.2) or, in other words, for $m=2$, there does not exist an equation of type (2.2) having as a solution the given function $V(t)$.

In Figure 2.2, a part of the same function $V(t)$, taken in the shorter interval $t \in(10,30)$, is presented. The corresponding phase plot $\vec{c}_{2}(t) \equiv$ $\{V(t), d V(t) / d t\}$ is presented in Figure 2.3. This time, there is no point of self-intersection, thus $\vec{c}_{2}(t)$ is a simple curve (even an elementary one). Moreover, in the next figures (Figures 2.4 and 2.5), the graphs of firstand second-time derivatives of $V(t)$ are presented. It is seen that simultaneous vanishing of the two derivatives has no place. (For example, in Figure 2.5, one of the points of self-intersection is under the abscissa. The same can be shown for the other points of self-intersection.) This means that the curve $\vec{c}_{2}(t)$ is both simple and regular in the interval $(10,30)$. Then, on the base of the above-formulated theorem, we can conclude that, in the interval $(10,30)$, the minimal order of equation of type (2.2), having $V(t)$ as a solution is $m=2$.

\section{Polynomial approximation of the right-hand side of the unknown differential equation}

Next, we want to approximate an unknown differential equation from a given numerical solution $y(t)$ that is near enough to the true analytic solution $V(t)$.

Here we propose an approximate procedure for determining unknown polynomial right-hand side of the differential equation. The procedure is based on the least squares method and the fact that we know with sufficient precision the values of $V(t)$ and its derivatives of an order equal to the equation order already defined by applying the above-mentioned theorems and classifications. In order to reconstruct (2.2), it can be written in 


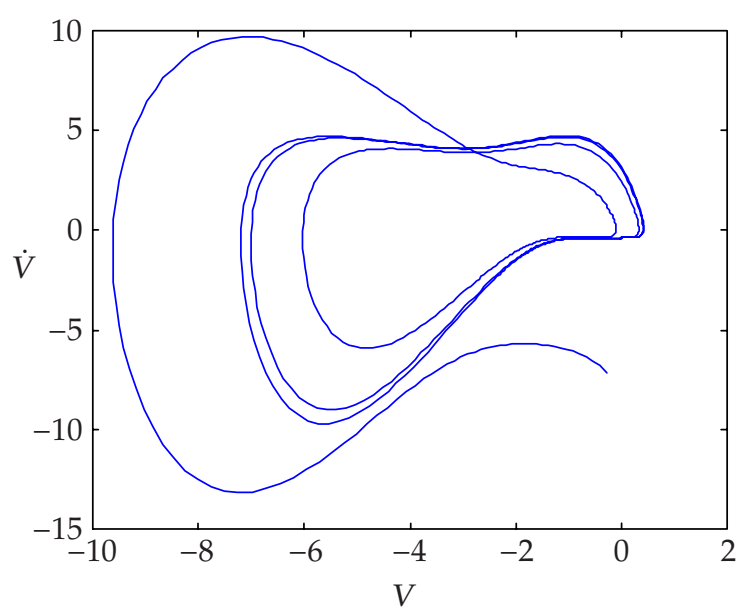

FIGURE 2.1. Phase plot of the action potential and its first derivative.

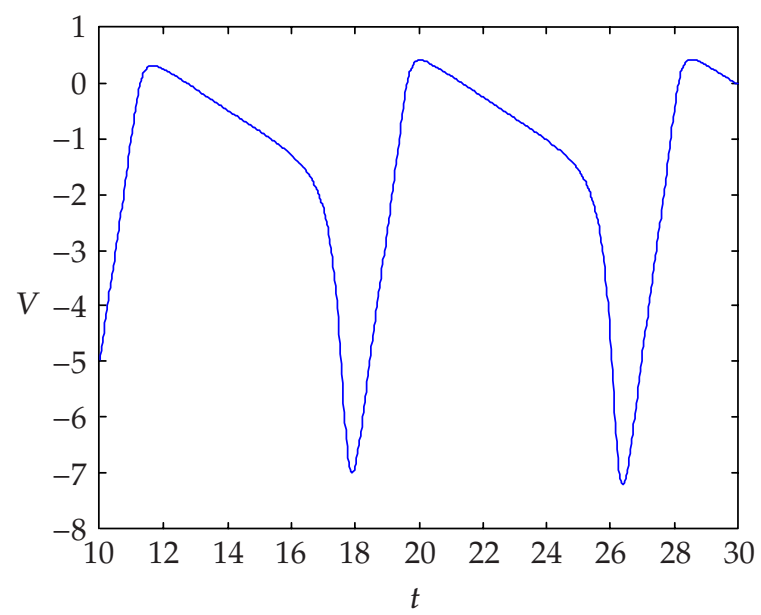

FIgURE 2.2. Action potential in the interval $t \in(10,30)$.

the form

$$
\begin{aligned}
\dot{V} & =V_{1}, \\
\dot{V}_{1} & =V_{2}, \\
& \vdots \\
\dot{V}_{m-1} & =V_{m} \\
\dot{V}_{m} & =P_{k}\left(V, V_{1}, V_{2}, \ldots, V_{m-1}\right),
\end{aligned}
$$




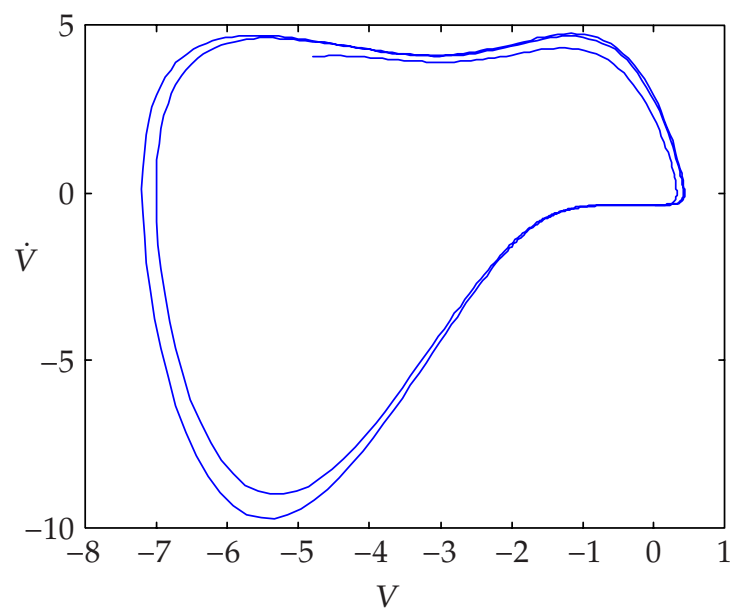

FIGURE 2.3. Phase plot of the action potential $V$ and its timederivative $\dot{V}$ in the interval $t \in(10,30)$.

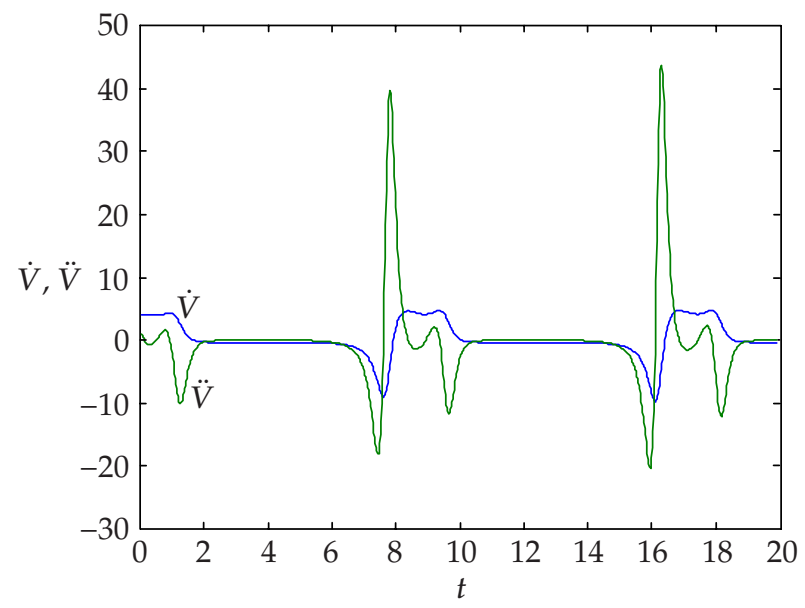

FIGURE 2.4. First $\dot{V}$ and second $\ddot{V}$ derivatives of the action potential.

where $P_{k}$ is a polynomial of sufficiently high power $k$ and $m$ is the unknown order of highest derivative in the equation. (In our case $m=2$ ). In more detail, the polynomial $P_{k}$ can be written in the form

$$
P_{k}\left(V, V_{1}, \ldots, V_{m-1}\right)=\sum_{l, l_{1}, \ldots, l_{m-1}=0}^{k} \xi_{l, l_{1}, \ldots, l_{m-1}} \prod_{j=1}^{m-1} V_{j}^{l_{j}}, \quad \sum l_{j} \leq k .
$$

Here $\xi_{l, l_{1}, \ldots, l_{m-1}}$ are the unknown polynomial coefficients. 


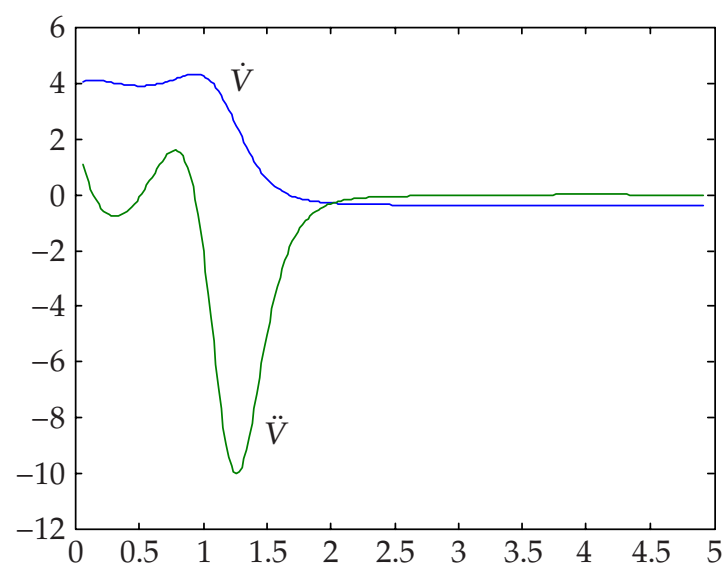

FIGURE 2.5. First and second derivatives near a point of self-intersection.

The problem of reconstruction is to find these coefficients under the condition that we know some time series $\left\{y\left(t_{i}\right)\right\}$ whose points are sufficiently near the points of the analytic time series $\left\{V\left(t_{i}\right)\right\}$. Thus, instead of (3.1), we can write the system

$$
\begin{aligned}
\dot{y} & =y_{1}, \\
\dot{y}_{1} & =y_{2}, \\
& \vdots \\
\dot{y}_{m-1} & =y_{m}, \\
\dot{y}_{m} & =P_{k}\left(y, y_{1}, y_{2}, \ldots, y_{m-1}\right)+\varepsilon\left(y, y_{1}, y_{2}, \ldots, y_{m-1}\right),
\end{aligned}
$$

where the polynomial $P_{k}$ has the same coefficients as (3.2) and $\varepsilon\left(y, y_{1}\right.$, $\left.y_{2}, \ldots, y_{m-1}\right)$ is a sufficiently small error function. Thus $\dot{y}=\dot{y}_{m}$ is a random variable. We can write a large number $N$ of values $\dot{y}_{i}(i=1, \ldots, N)$ of the random variable

$$
\dot{y}_{i}=P_{k}\left(y_{i}, y_{1 i}, \ldots, y_{m-1, i}\right)+\varepsilon_{i}=\sum_{l, l_{1}, \ldots, l_{m-1}=0}^{k} \xi_{l, l_{1}, \ldots, l_{m-1}} \prod_{j=1}^{m-1} y_{j i}^{l_{j}}+\varepsilon_{i},
$$

where $\varepsilon_{i}(i=1,2, \ldots, N)$ are sufficiently small random errors with normal (Gaussian) distribution. Certainly, this requirement of computational (or observational) noise is not necessary at all, but it is preferable. What is of essential importance here is the requirement for sufficient precision of the time series $\dot{y}_{i}(i=1,2, \ldots, N)$. This means it should be obtained 
by applying numerical differentiation (the well-known scheme of difference ratios) of an exact enough solution $y(t)$.

The relations (3.4) can be written in the form

$$
\varepsilon_{i}=\dot{y}_{i}-\sum_{l, l_{1}, \ldots, l_{m-1}=0}^{k} \xi_{l, l_{1}, \ldots, l_{m-1}} \prod_{j=1}^{m-1} y_{j i^{\prime}}^{l_{j}} \quad i=1,2, \ldots, N
$$

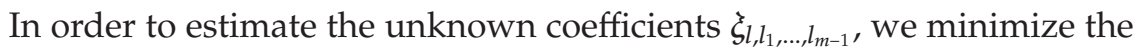
sum of error squares

$$
S=\sum_{i=1}^{N} \varepsilon_{i}^{2}
$$

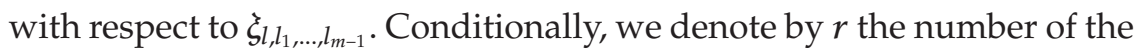
unknown coefficients. Then, to find the minimum of (3.6), we write $r$ equations in the form

$$
\frac{\partial S}{\partial \xi_{l, l_{1}, \ldots, l_{m-1}}}=-2 \sum\left(\dot{y}_{i}-\sum_{l, l_{1}, \ldots, l_{m-1}=0}^{k} \xi_{l, l_{1}, \ldots, l_{m-1}} \prod_{j=1}^{m-1} y_{j i}^{l_{j}}\right)=0
$$

The number of equations presented by (3.7) is equal to the number of unknowns. Thus, in principle, we can calculate the coefficients. For concrete values of polynomial power $k$ and order $m$ of the differential equation, the system (3.7) of $r$ linear algebraic equations for $r$ unknown coefficients can be presented in a corresponding normal form as it is the ordinary practice in the least squares method.

\section{Quantitative identification of GFN equation from a numerical solution of HHCC model}

We take Fitzhugh-Nagumo equation in the following generalized form:

$$
\begin{aligned}
\dot{y}= & y_{1} \\
\dot{y}_{1}= & w_{1}+w_{2} y+w_{3} y^{3}+w_{4} y^{5}+w_{5} y^{7}+w_{6} y_{1}+w_{7} y^{2} y_{1}+w_{8} y^{4} y_{1} \\
& +w_{9} y^{6} y_{1}+w_{10} y^{8} y_{1}+w_{11} y^{10} y_{1}+w_{12} y^{12} y_{1}+w_{13} y^{14} y_{1} \\
& +w_{14} y^{16} y_{1}+w_{15} y^{18} y_{1}+w_{16} y^{20} y_{1}+w_{17} y^{22} y_{1}+w_{18} y^{24} y_{1} \\
& +w_{19} y^{26} y_{1}+w_{20} y^{28} y_{1}+w_{21} y^{30} y_{1}+w_{22} y^{32} y_{1}+\varepsilon\left(y, y_{1}\right)
\end{aligned}
$$

Certainly, this is a particular case of the more general form (1.2). Applying the above-described least squares method to (4.1) for the given numerical data shown in Figures 2.2, 2.3, and 2.4, we obtain the following values 


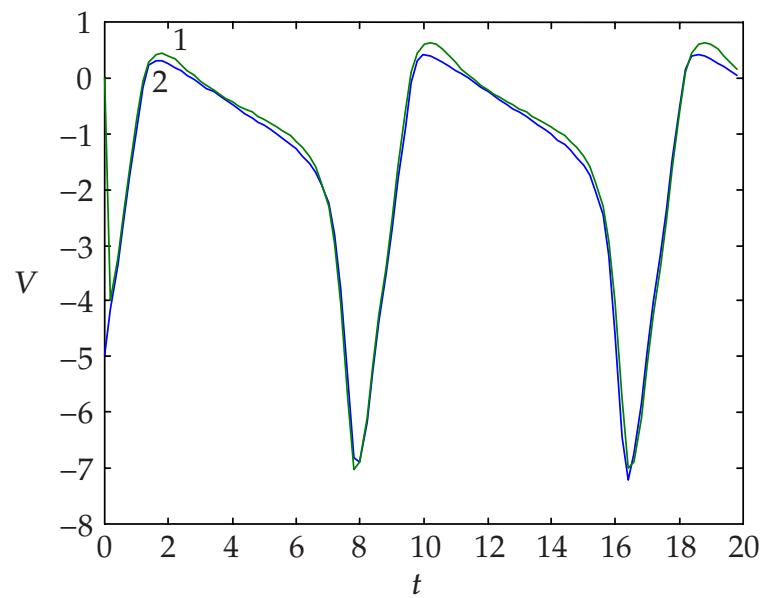

FIGURE 4.1. Reconstructed (1) and original (2) solutions for the action potential.

for the unknown coefficients $w_{i}(i=1,2, \ldots, 22)$ given consequently row by row in a long numerical format:

$$
\begin{array}{rr}
-1.264775993898694 \mathrm{e}+000 & -1.203737425127063 \mathrm{e}+000 \\
4.346112120853091 \mathrm{e}-001 & -1.880289825835857 \mathrm{e}-002 \\
1.768445286164994 \mathrm{e}-004 & -3.099982789537563 \mathrm{e}+000 \\
3.190076313652839 \mathrm{e}+000 & -6.176045130223027 \mathrm{e}-001 \\
-5.228009601502203 \mathrm{e}-002 & 3.196860016637852 \mathrm{e}-002 \\
-4.358331135826762 \mathrm{e}-003 & 2.757592982480551 \mathrm{e}-004 \\
-7.151909501225488 \mathrm{e}-006 & -1.040097178573921 \mathrm{e}-007 \\
1.185781356187376 \mathrm{e}-008 & -2.653411136153012 \mathrm{e}-010 \\
-3.530187474014420 \mathrm{e}-013 & 1.082689605526242 \mathrm{e}-013 \\
-1.462258253823620 \mathrm{e}-015 & -3.463614495001348 \mathrm{e}-018 \\
2.100520926807157 \mathrm{e}-019 & -1.239589912308053 \mathrm{e}-021
\end{array}
$$

After replacing these values for the coefficients in (4.1) and by solving (4.1) at the same initial conditions as they are in the original solution shown in Figure 2.2, we obtain a such named reconstructed solution shown in Figure 4.1, together with the given solution playing the role of an empirical one. It is seen that good enough fitting takes place between the two solutions.

\section{Conclusion}

The obtained results show that the formulated theorem and least squares method can be applied in principle to describe quantitatively action potential solutions of the empirical HHCC model by two-dimensional GFN 
equation. More exactly, we can do that for some almost periodic part of HHCC-potential solutions when the corresponding phase space reduces from dimension $m=4$ to lower dimension $m=2$. Thus we can conclude in this case that GFN equation is a quantitative and possibly a qualitative analog of HHCC model. Nevertheless, there is a possibility of applying a similar approach to enhance robustness of the identification. In other words, parametric system identification from a piece of a single trajectory for a single set of parameter values may not include enough information about the vector field of the underlying dynamical system, and the identification could fail in that case. This circumstance must be taken into consideration for future, more detailed qualitative analysis of GFN equations reconstructed quantitatively from experimental records of action potentials.

\section{Acknowledgment}

The author thanks Dr. P. Gospodinov and Dr. V. Petrov for helpful discussions.

\section{References}

[1] K. Doya and A. I. Selverston, Dimension reduction of biological neuron models by artificial neural networks, Neural Computation 6 (1994), no. 4, 696-717.

[2] R. Fitzhugh, Impulses and physiological states in theoretical models of nerve membrane, Biophys. J. 1 (1961), 445-466.

[3] A. L. Hodgkin and A. F. Huxley, The components of membrane conductance in the giant axon of Loligo, J. of Physiology 116 (1952), 473-496.

[4]_ Currents carried by sodium and potassium ions through the membrane of the giant axon of Loligo, J. of Physiology 116 (1952), 449-472.

[5]_ The dual effect of membrane potential on sodium conductance in the giant axon of Loligo, J. of Physiology 116 (1952), 497-506.

[6] A quantitative description of membrane current and its application to conduction and excitation in nerve, J. of Physiology 117 (1952), 500-544.

[7] A. L. Hodgkin, A. F. Huxley, and B. Katz, Measurement of current-voltage relations in the membrane of the giant axon of Loligo, J. of Physiology 116 (1952), 424-448.

[8] J. Keener and J. Sneyd, Mathematical Physiology, Interdisciplinary Applied Mathematics, vol. 8, Springer-Verlag, New York, 1998.

[9] T. B. Kepler, L. F. Abbott, and E. Marder, Reduction of conductance-based neuron models, Biol. Cybern. 66 (1992), no. 5, 381-387.

[10] J. S. Nagumo, S. Arimoto, and S. Yoshizawa, An active pulse transmission line simulating nerve axon, Proc. Inst. Radio Engineers 50 (1962), 2061-2070.

[11] V. Petrov, N. Georgiev, and J. Kurths, Determining phase space dimension of a dynamical system from analytic time series, J. Theoret. Appl. Mech. 32 (2002), no. 3, 13-28. 
[12] J. Rinzel, Excitation dynamics: insights from simplified membrane models, Fed. Proc. 44 (1985), 2944-2946.

Nikola V. Georgiev: Section of Biodynamics and Biorheology, Institute of Mechanics, Acad. G. Bonchev St., bl. 4, 1113 Sofia, Bulgaria

E-mail address: naksim@imbm.bas.bg 


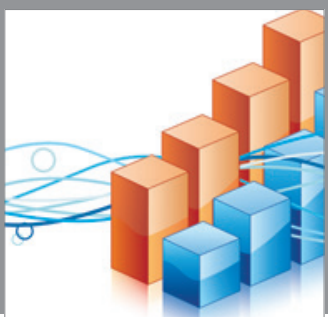

Advances in

Operations Research

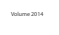

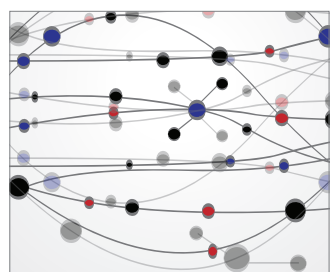

\section{The Scientific} World Journal
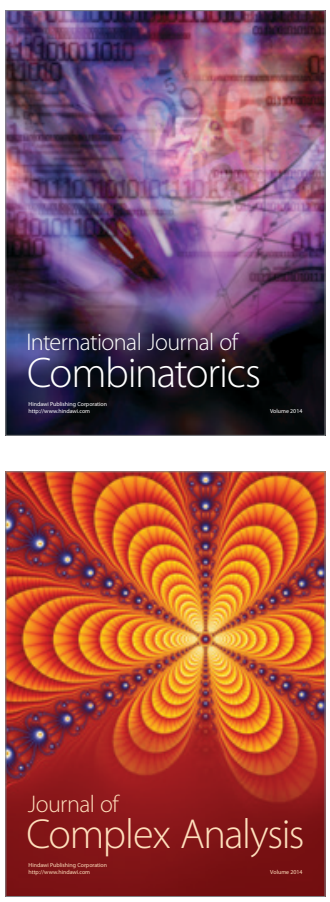

International Journal of

Mathematics and

Mathematical

Sciences
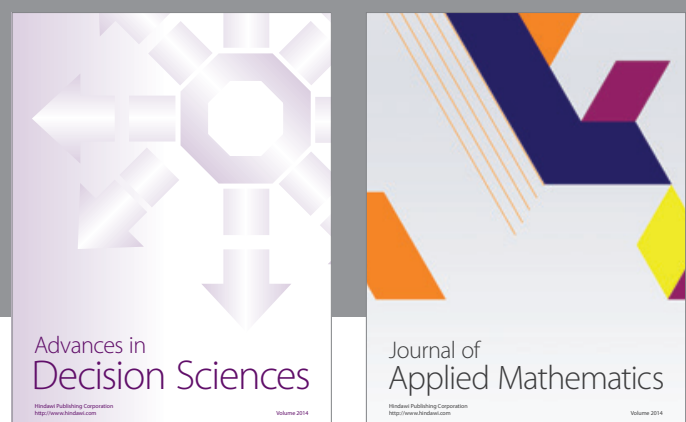

Journal of

Applied Mathematics
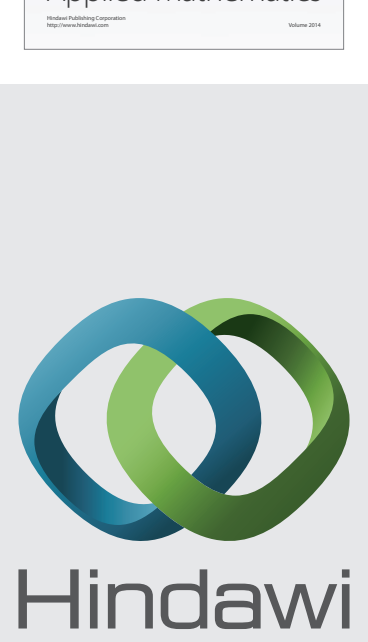

Submit your manuscripts at http://www.hindawi.com
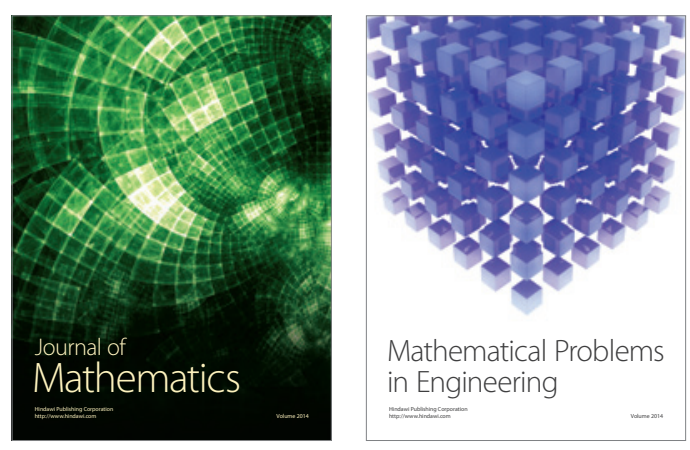

Mathematical Problems in Engineering
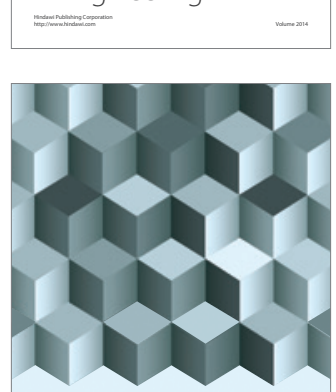

Journal of

Function Spaces
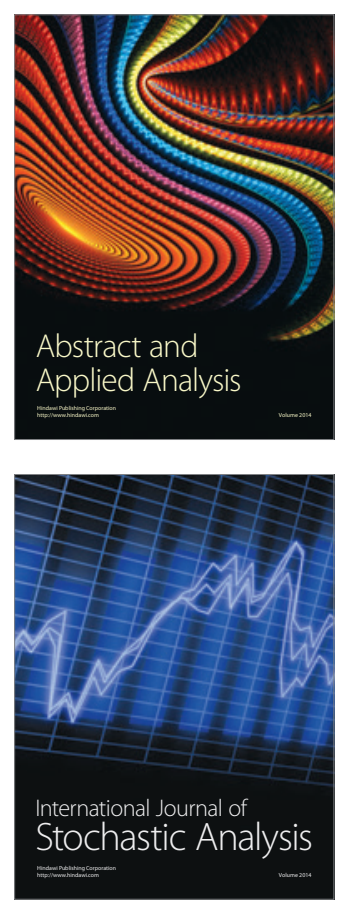

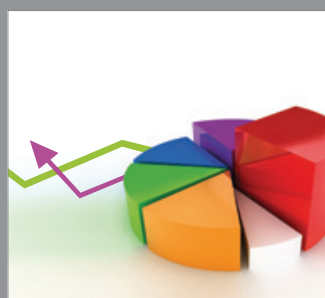

ournal of

Probability and Statistics

Promensencen
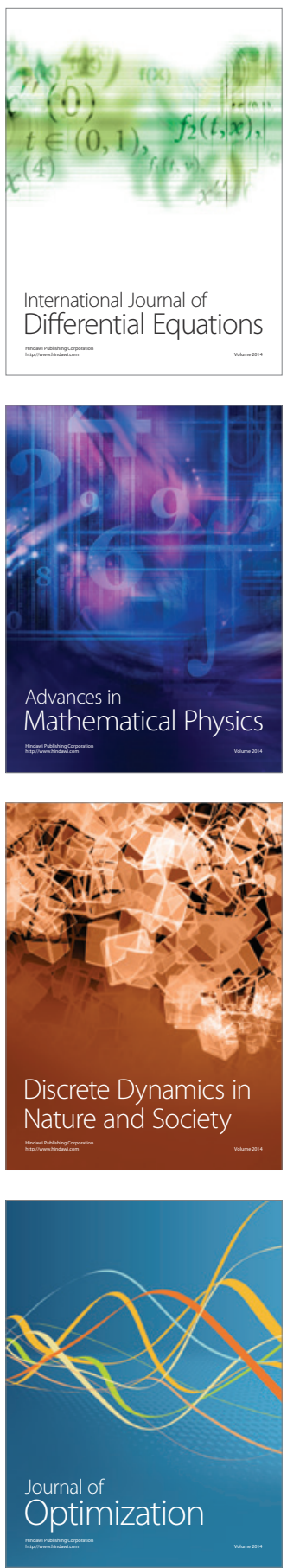